\title{
Detection of Cosmic-Ray Ensembles with CREDO
}

\author{
Krzysztof W. Woźniak ${ }^{1}$ for the CREDO Collaboration ${ }^{2, *}$ \\ ${ }^{1}$ Institute of Nuclear Physics, Polish Acedemy of Sciences Krakow, Poland \\ e-mail: krzysztof.wozniak@ifj.edu.pl \\ ${ }^{2}$ website: http://credo.science
}

\begin{abstract}
One of the main objectives of cosmic-ray studies are precise measurements of the energy and chemical composition of particles with extreme energies. Large and sophisticated detectors are used to find events seen as showers starting in the Earth's atmosphere with recorded energies larger than $100 \mathrm{EeV}$. However, a Cosmic-Ray Ensemble (CRE) developing before reaching the Earth as a bunch of correlated particles may spread over larger areas and requires an extended set of detectors to be discovered. The Cosmic-Ray Extremely Distributed Observatory (CREDO) is a solution to find such phenomena. Even simple detectors measuring the particle arrival time only are useful in this approach, as they are sufficient both to provide candidate CRE events and to determine the direction from which they are arriving.
\end{abstract}

\section{Introduction}

The primary ultra-high energy cosmic-ray (UHECR) particles arriving at the Earth are very rare as their number falls steeply with increasing energy. Those which interact strongly or electromagnetically do not reach the surface of the Earth, but instead initiate a cascade of secondary particles in the atmosphere. These secondary particles reach the ground as a shower of relatively low energy muons, electrons and photons and are detectable in cosmic-ray observatories. The most extended showers from a single cosmic-ray particle may, at ground level, have a diameter of 3-4 km [1]. The largest cosmic-ray observatories like Auger [2] and Telescope Array [3] have their detectors spread over very larger areas $\left(3000 \mathrm{~km}^{2}\right.$ and $700 \mathrm{~km}^{2}$, respectively) to ensure that such showers are fully contained. This coverage is however not sufficient in case the shower starts not at the top of the Earth atmosphere but at a significantly larger distance $[4,5]$. Correlated bunches of particles may be initiated by UHECR photons and decays of hypothetical heavy and energetic Dark Matter particles.

Simulations of Super pre-showers (SPS) initiated by a photon and starting near the Sun show that in such a scenario the secondary photons near the Earth would form a disk with a diameter of hundreds of $\mathrm{km}$ but with thickness of a few meters only [6]. In this case many photons arrive at almost the same time on top of the Earth atmosphere. Each of them has only a fraction of the energy of the primary photon, but still they may form numerous individual cascades. The energy of these cascades is at least an order of magnitude lower than that of the primary particle and

*The CREDO Collaboration: K. Almeida-Cheminant, Ł. Bratek, D.A. Castillo, N. Dhital, A.R. Duffy, D. Góra, B. Hnatyk, P. Homola, P. Jagoda, J. Jałocha-Bratek, M. Kasztelan, D. Lemański, P. Kovács, V. Nazari, M. Niedźwiecki, K. Smelcerz, K. Smolek, J. Stasielak, S. Stuglik, O. Sushchov, K.W. Woźniak, J. Zamora-Saá.

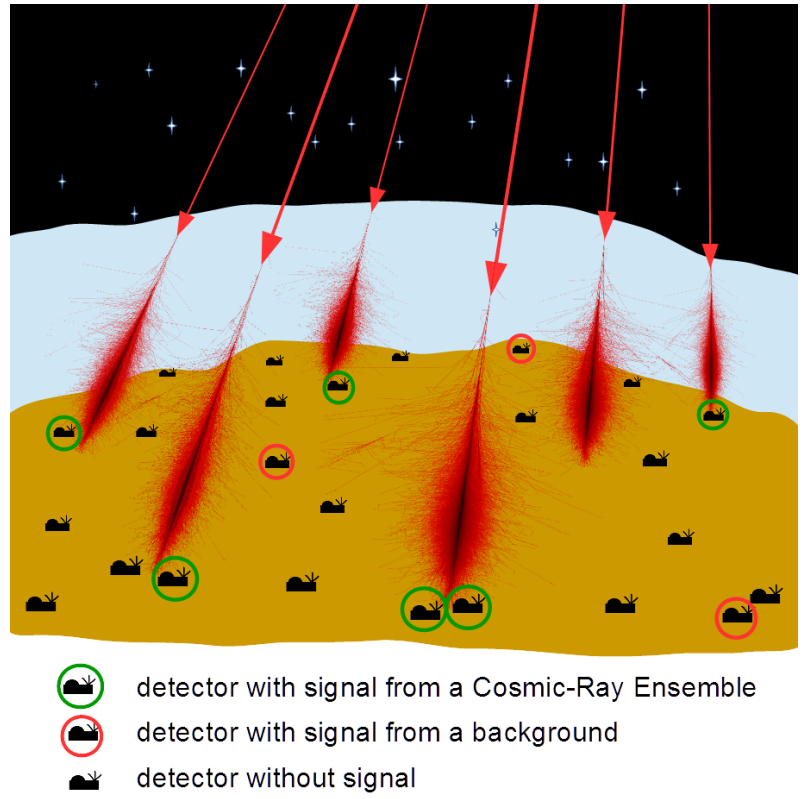

Figure 1. Detection of the Cosmic-Ray Ensemble (CRE) in distributed cosmic-ray detectors.

the distance between them may be a few km or more. Detection of such small showers, even if strongly correlated in time, by the traditional observatories like Auger may be impossible. If in the pre-shower only a few particles are created it is most probable that in the area covered by the observatory only one shower appears, and it would not differ from showers generated by a single cosmic ray. Several small showers may develop in the area covered by the observatory, if in the pre-shower the number of particles is much larger. However, in this case the energy of these 


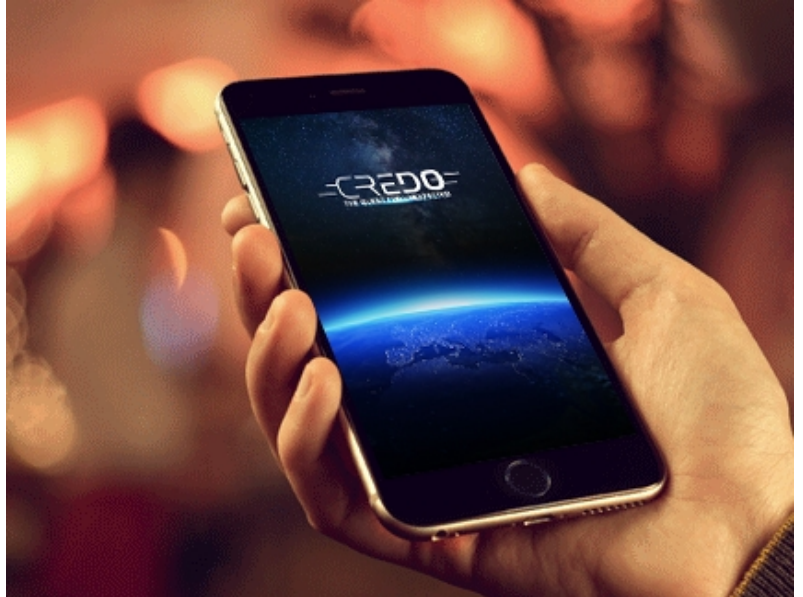

Figure 2. A smartphone with the CREDO application.

particles has to be relatively small, thus the signals in the detectors may have a pattern too different from a typical high energy cascade to trigger recording of the event.

\section{Cosmic-Ray Extremely Distributed Observatory}

Particles from a Cosmic-Ray Ensemble may arrive in places hundreds or even thousands $\mathrm{km}$ distant and produce many individual cascades. Their signature would be the coincidence in time. Consequently, the detectors used to register them need to cover the largest area possible, but may be quite sparse. It is not necessary to register many signals from individual cascades, it's better to detect signals from several different cascades, even if it would be a single measurement for each cascade. The best results can be achieved when the information from detectors spread over a country, continent or even the whole Earth are collected and analyzed together. The idea of CRE detection is presented in Figure 1. The individual cascade leaves a signal in one or a few detector stations. There are stations in which no signal is registered in the analyzed time interval and also stations with a background signal - for example cosmic rays not connected with CRE or from decays of radioactive nuclei.

The detectors registering the arrival time of particles are sufficient for testing the coincidences. However, rejection of backgrounds play an important role in extraction of the signal and measurements of CRE properties described in Section 3. Assuming that CRE initiates several small cascades it's reasonable to require a hardware based local coincidences of individual detectors within a station or a coincidence of nearby stations. This way contribution from uncorrelated background signals (mainly radioactive decays) can be suppressed. It does not solve the problem of small cascades from cosmic rays not belonging to the Cosmic-Ray Ensemble - but their contribution, at least partially, can be removed in the analysis.

The minimal hardware requirements are so easy to fulfill that the simplest of detectors capable of registering the time of a passage of a particle from a cosmic shower can
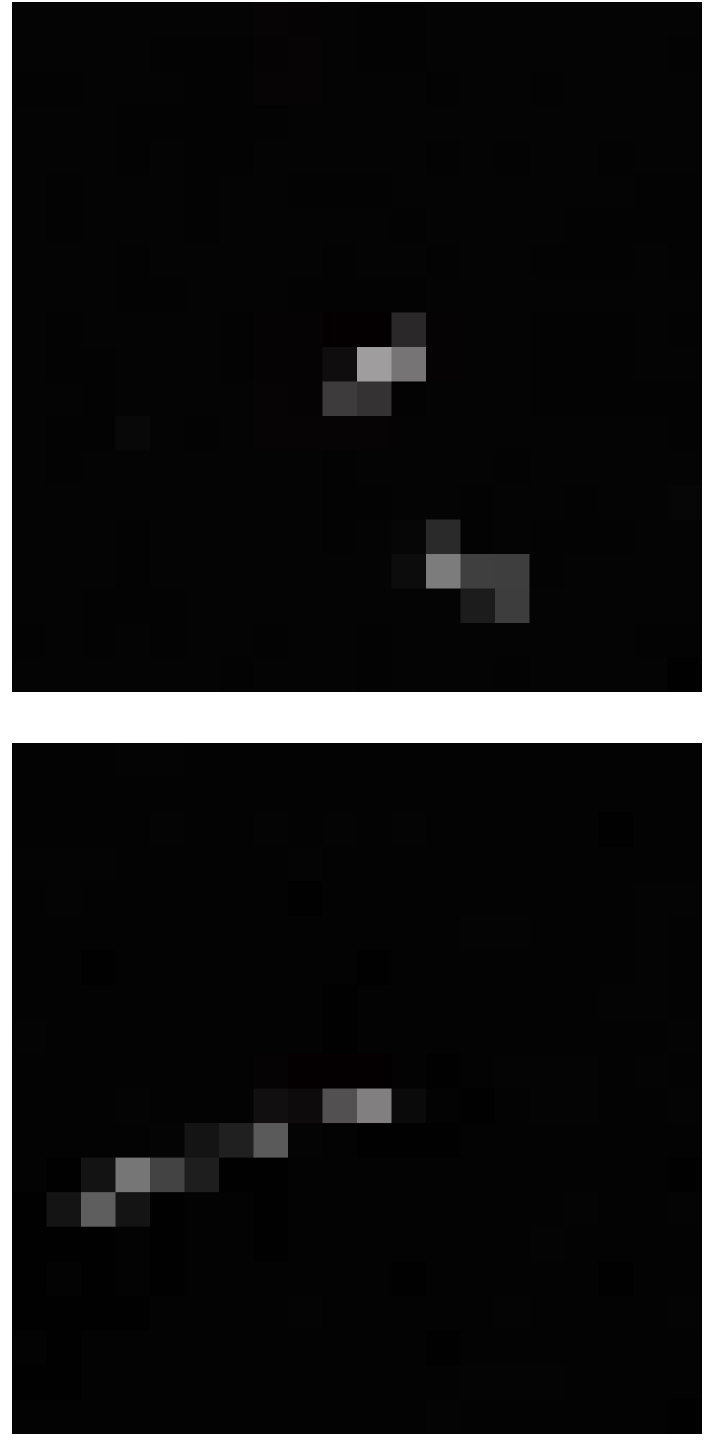

Figure 3. Examples of images taken with the completely covered camera of a smartphone using the CREDO application.

be useful. The most popular of such devices is a smartphone equipped with a camera and a GPS module. It can work as a cosmic-ray detector if the camera lens is covered (to prevent external light) and an appropriate application is running.

The idea of using smartphones to detect cosmic rays is not new. There are two groups CRAYFIS [7, 8] and DECO $[9,10]$ which developed their custom applications for this purpose for several years. However, their software is proprietary thus the Open Source CREDO application (Figure 2) was also created [11].

The CREDO application works when the smartphone is attached to a power supply (to prevent discharging of the battery) and the camera is protected from light. The pictures similar to those presented in Figure 3 are constantly taken and those with a pattern ressembling that from a cosmic ray are sent to the server over the Internet. The information from the smartphones is stored and a summary of the activities is provided in a WWW page [12]. Currently, 


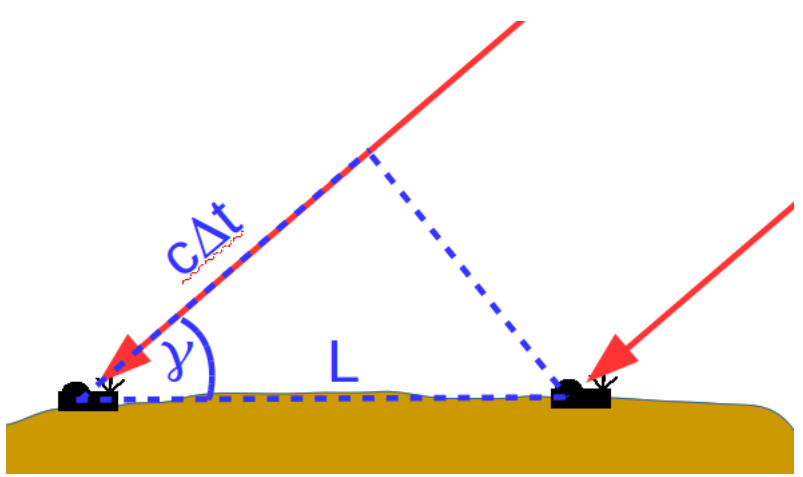

Figure 4. Estimation in two dimensions of the direction of an incoming Cosmic-Ray Ensemble on the basis of position and time registration in two detectors.

the work on prototypes of simple and inexpensive desktop type cosmic-ray detectors is also ongoing.

\section{Time Based Triangulation}

The Cosmic-Ray Ensemble created at a point very far from the Earth arrives as a front of almost parallel moving particles. In this case a simple measurement of the position of the detector and the time when such a particle arrives is sufficient to determine the direction of the front, as shown in Figure 4. Assuming the detected particles are traveling with the speed of light, $c$, the registration of the times $t_{1}$ and $t_{2}$ in two detectors which are placed at a distance $L$, enables the calculation of the inclination of the front, $\gamma$, as:

$$
\cos (\gamma)=c\left(t_{1}-t_{2}\right) / L .
$$

This angle calculated in two dimensions is in fact a half of the apex angle of the cone that is formed by all possible directions of the front. Two detectors (stations) are not sufficient to determine the direction of the front in 3D. However it is enough to add a third station with a signal, to construct two cones of possible directions of the front, with a common apex. Then there are two lines - intersections of the cones - and that of them which is pointing above the ground is the correct one. This way even only three station with signals from CRE with a flat front after relatively simple calculations of the Time Based Triangulation (TBT) method allow the estimation of the direction of the CRE. However, in the presence of a background signal in even one of these stations the results of calculations are severely distorted. Frequently, the estimated cones may have no intersection, either because a small cone is inside a bigger one or the two small cones are separated. In most cases, however, TBT calculations give a direction differing greatly from the correct one. It is thus necessary to test all combinations of three stations and analyze calculated directions to find the CRE direction.

\section{Simple CRE simulation}

The accuracy of calculations in the Time Based Triangulation method was tested using simple simulations of a
Super-preshower starting very far from the Earth and thus approximated by a flat front of particles. The positions of hypothetical detectors are randomly generated in the area approximately representing the territory of USA. The exact arrival time is randomly smeared with a width of $1 / 3000000 \mathrm{~s}$, an uncertainty coresponding to the $1 \mathrm{~km}$ position error along the SPS direcion. Signals from the SPS is simulated always in 5 stations, but in addition in some number of other stations background signals are randomly generated in the time interval around the arrival time of the SPS. In Figure 5 results of TBT reconstruction for one event in which SPS signals and background are present in 5 and 10 stations, respectively, are shown. The calculations were performed for all $15 ! /(3 !(15-3) !)=455$ combinations of three stations with signals, but only in 5!/(3!(5$3) !)=10$ combinations in all selected stations SPS signal is recorded. Even if one station contains a signal from background the result of TBT calculations can be completly wrong. In the grid of geographical coordinates (Figure 5 upper plot) many calculated directions are thus false and are spread over almost a half of the solid angle. In a closer examination of the angular area more focused on the real direction of SPS we can see an evident cluster of results near the correct direction and a less dense population around it. The calculated values from real signals are quite precise, the errors do not exceed $1^{\circ}$ thus for small and moderate background fractions the cluster at the SPS directions should be easy to identify.

For the case of 5 real and 10 background signals presented in Figure 5 the correct SPS direction can be guessed after checking the plot "by eye", but in more difficult cases much more elaborate data analysis is necessary. In simulations with smaller number of stations with a background the density of false directions is lower and the correct direction is more pronounced. For larger number of stations with backgrounds the cluster showing the correct direction becomes less visible and may be difficult to find. It is thus very important to keep the ratio of stations with real signal to those with a background signal as high as possible.

\section{Summary}

An obvious extension to the measurements of cosmic-ray showeres initiated by a single high energy particle are studies of Cosmic-Ray Ensembles. They may give an opportunity to discover new phenomena including new types of interactions or decays of massive particles. The purpose of CREDO is to collect all available cosmic-ray data and analyze correlations of signals in detectors spread over large distances. In this case detectors as simple as smartphones may be useful, thus an Open-source CREDO application was created. For measurements of electromagnetic Super-preshowers information from even small tabletop detectors may be sufficient. The Time Based Triangulation method allows calculation of direction of a SPS if only the background rate is not too large. 

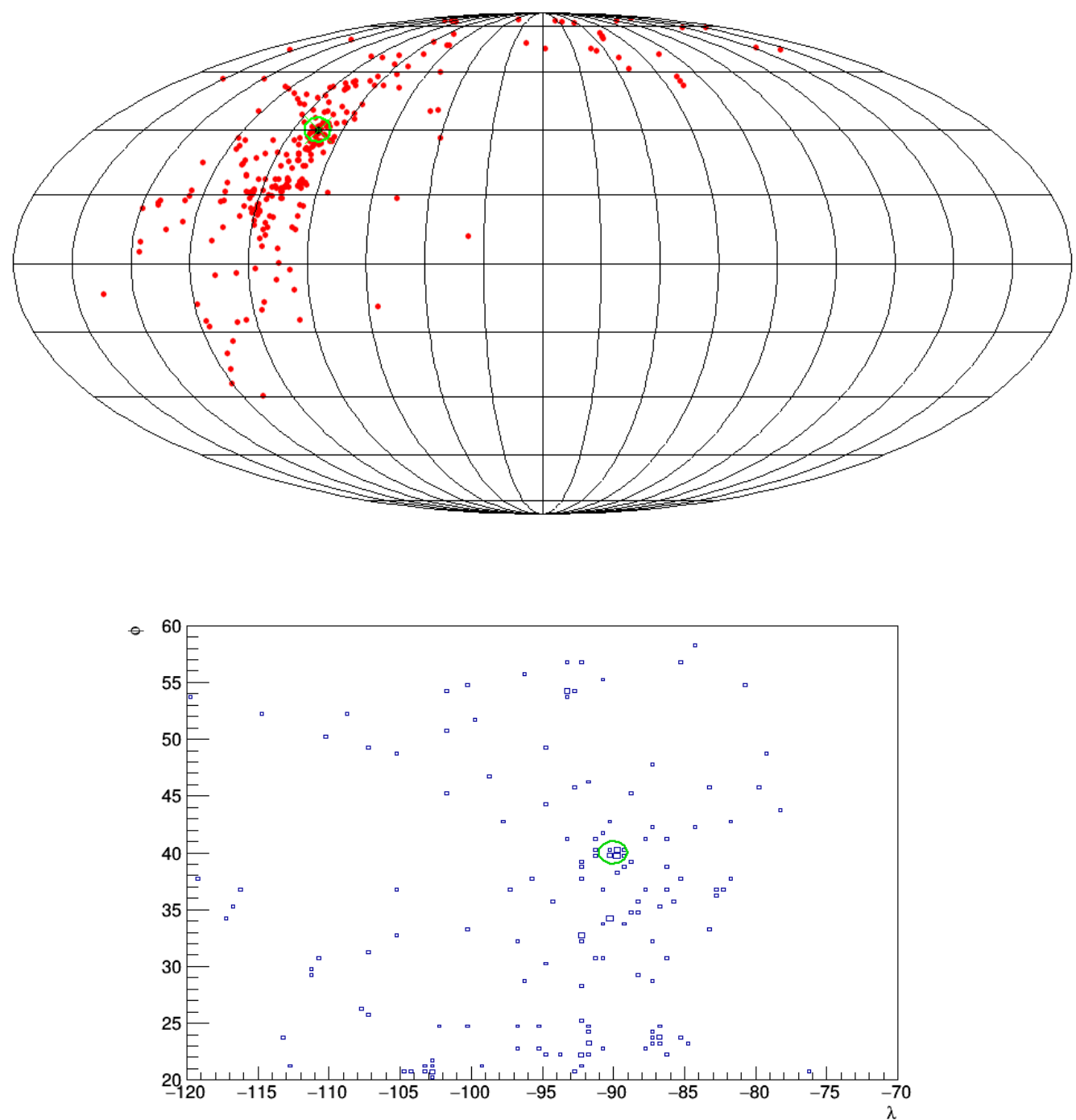

Figure 5. Estimation of possible directions of an incoming Cosmic-Ray Ensemble using the Time Based Triangulation method. Simulated signals from CRE were detected in 5 stations while in another 10 stations randomly generated background signals are present. In the upper plot all estimated directions in geographic coordinates system are shown in Mollweide projection. The correct CRE direction is denoted by the green circle, red points represent false estimates, obtained from calculations using at least one background signal. In the lower plot for selected range of latitude, $\phi$, and longitude, $\lambda$, the calculated directions are shown as boxes, the correct ones are in the green circle.

\section{Acknowledgments}

This research has been supported in part by the Governments of Czechia, Hungary, Poland and Slovakia through Visegrad Grant 21720040 from International Visegrad Fund and by the PLGrid Infrastructure. We thank the staff at ACC Cyfronet AGH-UST for their helpful support. CREDO application is developed in Cracow University of Technology.

\section{References}

[1] K. Kampert, A.A. Watson, arXiv:1207.4827 [physics.hist-ph] (2012).

[2] The Pierre Auger Collaboration, Nucl. Instr. Meth. A 798 (2015) 172.

[3] T. Abu-Zayyad, et al. Nucl. Instr. Meth. A 689 (2012) 87.
[4] T. Erber, Rev. Mod. Phys. 38 (1966) 626.

[5] B. McBreen and C. J. Lambert, Phys. Rev. D24 (1981) 2536.

[6] N. Dhital, et al. (CREDO Collaboration), PoS (ICRC2017) 1078 (arXiv:1709.05196 [astro-ph.IM]).

[7] https://crayfis.io

[8] D. Whiteson, M. Mulhearn, C. Shimmin, K. Cranmer, K. Brodie, D. Burns (CRAYFIS), arXiv:1410.2895 [astro-ph.IM].

[9] https://wipac.wisc.edu/deco/home

[10] J. Vandenbroucke et al. (DECO), PoS (ICRC2015) 691 (arXiv:1510.07665v1 [astro-ph.IM]).

[11] https://credo.science/credo-detector-mobile-app/

[12] https://api.credo.science/web/ 